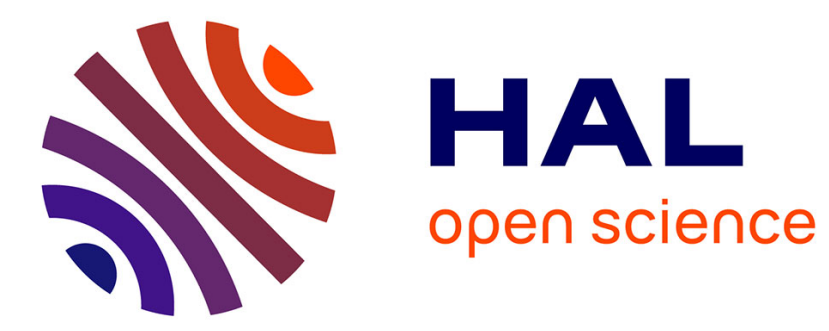

\title{
Non-Linearity Extremum in Niobium Doped Potassium Tantalate
}

O. Bidault, Mario Maglione

\section{To cite this version:}

O. Bidault, Mario Maglione. Non-Linearity Extremum in Niobium Doped Potassium Tantalate. Journal de Physique I, 1997, 7 (3), pp.543-552. 10.1051/jp1:1997106 jpa-00247342

HAL Id: jpa-00247342

https://hal.science/jpa-00247342

Submitted on 1 Jan 1997

HAL is a multi-disciplinary open access archive for the deposit and dissemination of scientific research documents, whether they are published or not. The documents may come from teaching and research institutions in France or abroad, or from public or private research centers.
L'archive ouverte pluridisciplinaire HAL, est destinée au dépôt et à la diffusion de documents scientifiques de niveau recherche, publiés ou non, émanant des établissements d'enseignement et de recherche français ou étrangers, des laboratoires publics ou privés. 


\title{
Non-Linearity Extremum in Niobium Doped Potassium Tantalate
}

\author{
O. Bidault $\left({ }^{*}\right)$ and M. Maglione
}

Laboratoire de Physique, Université de Bourgogne $\left({ }^{* *}\right)$, BP 138, 21004 Dijon, France

(Received 29 July 1996, revised 16 October 1996, accepted 20 November 1996)

PACS.64.60.-i - General studies of phase transitions
PACS.77.22.Ch - Permittivity (dielectric function)
PACS.77.80.-e - Ferroelectricity and antiferroelectricity

\begin{abstract}
We report non-linear dielectric measurements in the $\mathrm{KTaO}_{3}: \mathrm{Nb}$ system $\left(x_{\mathrm{Nb}} \leq 0.05\right)$. The non-linear susceptibility diverges at low temperatures and reaches maximum values in the intermediate range $\left(0.0075 \leq x_{\mathrm{Nb}} \leq 0.02\right)$. To account for this extremum non linearity, we propose a model of temperature dependent clusters which fits with a number of experimental reports. We also suggest that this model may be applied to the related compounds $\mathrm{SrTiO}_{3}: \mathrm{Ca}$ and $\mathrm{KTaO}_{3}: \mathrm{Na}$.
\end{abstract}

\section{Introduction}

The system of interest here, potassium tantalate niobate $\mathrm{KTa}_{1-x} \mathrm{Nb}_{x} \mathrm{O}_{3}$ (or KTN), is a simple perovskite lattice $\left(\mathrm{KTaO}_{3}\right)$ containing a dilute concentration of isoelectronic defects $\left(\mathrm{Nb}^{5+}\right)$. By varying the $\mathrm{Nb}$ concentration, the properties can be adjusted between those of $\mathrm{KNbO}_{3}$ and $\mathrm{KTaO}_{3}$. Solid solutions of the incipient ferroelectric $\mathrm{KTaO}_{3}$ with $\mathrm{KNbO}_{3}$ are known to exhibit complex polar behavior at low temperatures.

Potassium niobate behaves as a ferroelectric undergoing a sequence of ferroelectric phase transitions with structural changes of the type cubic - quadratic - orthorhombic - rhombohedral on cooling [1]. On the other hand pure potassium tantalate is an incipient ferroelectric whose dielectric constant increases rapidly as the temperature is reduced following a modified Curie law $\varepsilon_{1} \approx C / T^{\gamma}[2]$. The increase of the dielectric susceptibility is related to the softening of the ferroelectric phonon mode which is stabilized at low temperatures by quantum fluctuations: consequently the crystal does not undergo a phase transition and the lattice remains cubic at all temperatures.

The substitution of $\mathrm{Nb}$ for Ta yields mixed crystals which exhibit a paraelectric to ferroelectric structural phase transition provided $x_{\mathrm{Nb}}$ is high enough. In fact the lattice properties of the $\mathrm{KTa}_{1-x} \mathrm{Nb}_{x} \mathrm{O}_{3}$ system are strongly dependent on the $\mathrm{Nb}$ content: for $x_{\mathrm{Nb}} \leq 0.0075$, KTN remains paraelectric while it undergoes a ferroelectric distortion at temperatures directly proportional to the substitutional rate for $x_{\mathrm{Nb}} \geq 0.02$. Until $x_{\mathrm{Nb}}=0.05$ only a large increase of the dielectric constant is reported. This dielectric peak is usually associated with a cubicrhombohedral symmetry change [3]. In the intermediate range $0.0075 \leq x_{\mathrm{Nb}} \leq 0.02$ the situation is less clear and some glass-like properties are suggested by several experiments: dielectric

$\left.{ }^{*}\right)$ Author for correspondence (e-mail: maglione@ satie.u-bourgone.fr)

$\left({ }^{* *}\right)$ UPRESA CNRS 5027

(C) Les Éditions de Physique 1997 
relaxation [4], linear birefringence [5], refractive index [5], inelastic light [6] and inelastic neutron scattering [7] experiments. Moreover high resolution X-ray diffraction measurements [8] show no detectable change in symmetry down to $10 \mathrm{~K}$ whereas a $\mathrm{Nb}$ dependent minimum in the cubic unit cell volume is reported. This glass-like behavior was originally observed by Samara [4] in a KTN $x_{\mathrm{Nb}}=0.009$ sample. From a dielectric dispersion in the kilohertz range under hydrostatic pressure, he concluded against a ferroelectric phase transition and in favor of the freezing of slow relaxing clusters. The direction of the spontaneous polarization, as well as NMR measurements, indicate that the dominant $\mathrm{Nb}$ displacement is along [111]. In fact, magnetic resonance data [9] show that the polar objects in KTN are not single $\mathrm{Ta}^{5+}$ or $\mathrm{Nb}^{5+}$ off-center ions, but coherent polar regions of about 100 unit cells size. The coexistence of short range clusters and long-range ferroelectricity was subsequently demonstrated on the basis of Raman scattering $[10,11]$. A detailed description of clusters in KTN was given in a review by Vugmeister and Glinchuk [12].

It is the scope of our report to show that non-linear dielectric experiments are a useful tool to probe the existence of polarized clusters even for very low $\mathrm{Nb}$ contents. Such experiments under dc fields were already performed in KTN. Metastability was evidenced on the basis of the isothermal relaxation of $\varepsilon_{\text {Linear }}\left(\varepsilon_{\mathrm{L}}\right)$ following the switching of the dc field [13]. This was explained by the growth of quadrupolar domain state. Such state was also inferred from polarization hysteresis loops [14]. In the present study, the data were recorded at temperatures and fields away from the metastability and irreversibility regime. In this sense, we probed the precursor effects of the low temperature domain state.

Non-linear susceptibility $\left(\varepsilon_{\mathrm{NL}}\right)$ experiments have been already performed in a $0.009 \mathrm{Nb}$ doped crystal. These revealed a non-analytic variation of the dielectric susceptibility which was attributed to a dipolar glass state [15]. In order to extend the investigated niobium content to lower and higher concentrations $\left(0 \leq x_{\mathrm{Nb}} \leq 0.05\right)$, we measured $\varepsilon_{\mathrm{NL}}$ in five KTN samples but in a different way that the one described in reference [15]. In the high temperature phase, we find that $\varepsilon_{\mathrm{NL}}$ depends critically on temperature and reaches maximum values in the range $0.0075 \leq x_{\mathrm{Nb}} \leq 0.02$, giving evidence of disorder. We thus compare our results with similar dielectric random systems in which non-linearity has already been reported: $\mathrm{K}_{1-x} \mathrm{Na}_{x} \mathrm{TaO}_{3}[16$, 17] and $\mathrm{Sr}_{1-x} \mathrm{Ca}_{x} \mathrm{TiO}_{3}[18-20]$.

\section{Experiment}

Our experiments were carried out on five different single-crystals grown by L.A. Boatner: a pure $\mathrm{KTaO}_{3}$ ("ultra high purity") and four niobium doped samples $\left(x_{\mathrm{Nb}}=0.0075, x_{\mathrm{Nb}}=0.015\right.$, $x_{\mathrm{Nb}}=0.02$ and $x_{\mathrm{Nb}}=0.05$ ). They were cut in the form of thin plates $\approx 6 \mathrm{~mm}^{2}$ in area and $\approx 1 \mathrm{~mm}$ thick. Vacuum-deposited gold films served as electrodes on the large (100) faces.

The samples were placed into a cryogenic environment with a long-term temperature stability of $10 \mathrm{mK}$. The dielectric susceptibility was recorded using either an HP 4192 or an HP 4284 impedance analyzer at fixed temperatures $(4<T<100 \mathrm{~K})$ and $f_{\text {mes }}=100 \mathrm{kHz}$. In the same time an applied dc field, $E$, was ranging from 0 up to $25 \mathrm{kV} / \mathrm{m}$. Obviously, the measuring frequency has been chosen so that the dispersion at $f<f_{\text {mes }}$ was negligible for all field while keeping a good precision on the recorded data. For all non-linear experiments, we took care that the measuring ac signal was much smaller than the superimposed dc bias in order to avoid any mixing of non-linearities. We also made sure of reversibility by cycling the dc bias between zero and its maximum value as exposed below. This restricted the study to the high temperature phase.

This experimental process is different from the one used by Lyons et al. [15]. Indeed they determined non-linearity by relating $\varepsilon_{\mathrm{NL}}$ to the harmonic amplitudes generated by a sinusoidal 


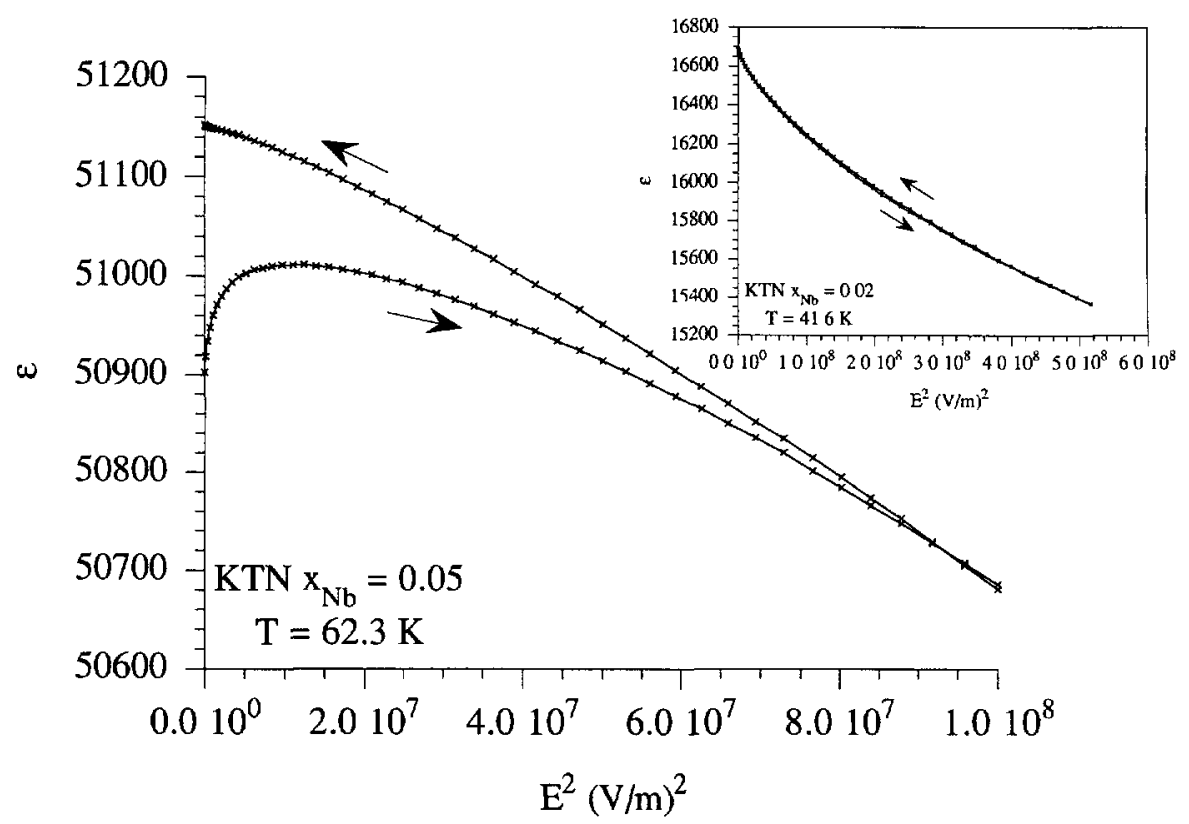

Fig. 1. - Dielectric susceptibility versus the squared dc electric field in $\mathrm{KTa}_{1-x} \mathrm{Nb}_{x} \mathrm{O}_{3}\left(x_{\mathrm{Nb}}=0.05\right)$ at a fixed temperature in the non-polar phase. The data were recorded during scanning $E$ from 0 to $E_{\max }$, then from $E_{\max }$ to 0 . This cycle shows the irreversibility at such a high $\mathrm{Nb}$ doping. Insert: the same experiment in a $\mathrm{KTa}_{1-x} \mathrm{Nb}_{x} \mathrm{O}_{3}\left(x_{\mathrm{Nb}}=0.02\right)$ crystal. This time, the reversibility appears clearly.

signal $V_{0} \sin \omega t$. In this manner, the measurements were made by increasing the ac voltage amplitude $\left(V_{0}\right)$ whereas in our study we look at the dc field $(E)$ dependence of the first order harmonic response.

\section{Results}

As earlier explained we have systematically recorded the dielectric susceptibility versus the surimposed dc field as it was increasing from 0 to $E_{\max }$ then decreasing down to 0 . For all the investigated samples, except for the more doped one, a clear reversibility is observed for all the temperatures in the non-polar phase (insert, Fig. 1). On the other hand, the KTN $x_{\mathrm{Nb}}=0.05$ exhibits a complex behavior with irreversibility even at temperatures much higher than $T_{\max }$, the temperature at which $\varepsilon$ peaks (Fig. 1). In this case the $\varepsilon_{\mathrm{NL}}$ determination is ambiguous. Thus, in the following, we will focus on the more dilute samples $\left(x_{\mathrm{Nb}} \leq 0.02\right)$, whereas the fitted values deduced for $x_{\mathrm{Nb}}=0.05$ are only indicative.

In a first step, we turn to a description of our results obtained in the KTN $x_{\mathrm{Nb}}=0.02$ crystal.

From the shape of the curve $\varepsilon\left(E^{2}\right)$ (insert, Fig. 1), it is obvious that $\varepsilon$ is an analytic function of field. The dielectric susceptibility may be developed in terms of even powers of the dc electric field,

$$
\varepsilon(E, T)=\varepsilon_{\mathrm{L}}(T)-\varepsilon_{\mathrm{NL}}(T) E^{2}+\varepsilon_{\mathrm{NL}}^{*}(T) E^{4}-O\left(E^{6}\right) .
$$

In this equation, $\varepsilon_{\mathrm{L}}$ stands for the linear susceptibility, $\varepsilon_{\mathrm{NL}}$ and $\varepsilon_{\mathrm{NL}}^{*}$ are respectively the first and second non-linear terms. Equation (1) allows to fit the data in all the investigated electric 


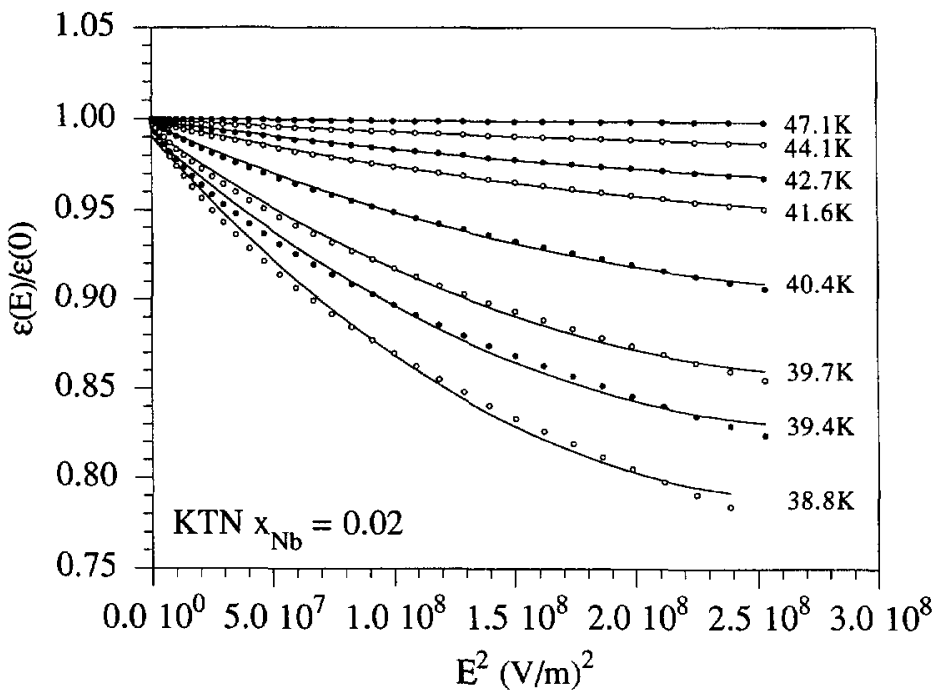

Fig. 2. - Normalized dielectric susceptibility as a function of the squared dc electric field in a $\mathrm{KTa}_{1-\gamma} \mathrm{Nb}_{x} \mathrm{O}_{3}\left(x_{\mathrm{Nb}}=0.02\right)$ crystal at different temperatures. The solid lines result from the best fit using (1) on the absolute variation of $\varepsilon(E)$.

field range with a good accuracy. This can be seen in Figure 2, which displays $\varepsilon$ in a normalized diagram as a function of $E^{2}$ at different temperatures $\left(T \geq T_{\max }=38 \mathrm{~K}\right)$. The second-order term $\varepsilon_{\mathrm{NL}}$ is thus deduced from the best fit shown by the solid lines. This value is connected with the initial slope at low fields whereas the $E^{4}$ contribution $\left(\varepsilon_{\mathrm{NL}}^{*}\right)$ becomes dominant for much higher fields and is related to the subsequent curvature. We will restrict our interest to the first non-linear term and especially to its temperature variation.

Indeed Figure 2 shows that the non-linear behavior decreases when the temperature is raised and finally vanishes at about $15 \mathrm{~K}$ above $T_{\max }$. This evolution is more clearly illustrated in Figure 3. All the $\varepsilon_{\mathrm{NL}}$ values deduced from equation (1) are reported on a logarithmic scale as a function of temperature for $x_{\mathrm{Nb}}=0.02$ (closed circles) together with the other studied samples. The $\varepsilon_{\mathrm{NL}}$ susceptibility diverges at a temperature close to $T_{\max }(38 \mathrm{~K})$. The divergence of the susceptibility is expected to follow in the high temperature phase $\left(T>T_{\max }\right)$ :

$$
\begin{aligned}
\varepsilon_{\mathrm{L}} & =\frac{K_{\mathrm{L}}}{\left(T-T_{\mathrm{c}}\right)^{\gamma L}} \\
\varepsilon_{\mathrm{NL}} & =\frac{K_{\mathrm{NL}}}{\left(T-T_{\mathrm{c}}\right)^{\gamma \mathrm{NL}}} .
\end{aligned}
$$

The well known Curie-Weiss law can be deduced from equation (2a) by setting $\gamma_{\mathrm{L}}=1$. As explained in reference [18], the fitted values (the critical temperature and exponents) are very sensitive to the selected data. This problem can be overcome by plotting $\varepsilon_{\mathrm{NL}}$ versus $\varepsilon_{\mathrm{L}}$ which leads to a power law,

$$
\varepsilon_{\mathrm{NL}} \propto\left(\varepsilon_{\mathrm{L}}\right)^{\nu} \quad \text { with } \nu=\frac{\gamma_{\mathrm{NL}}}{\gamma_{\mathrm{L}}}
$$

In the Landau theory, $\nu$ is expected to be equal to 4 for a true ferroelectric phase transition. Experimentally $\nu$ can be calculated from the slope of the curve $\varepsilon_{\mathrm{NL}}$ versus $\varepsilon_{\mathrm{L}}$ in a $\log -\log$ scale (Fig. 4). For $x_{\mathrm{Nb}}=0.02$, the fit (over four orders of magnitude on $\varepsilon_{\mathrm{NL}}$ ) leads to a value close 


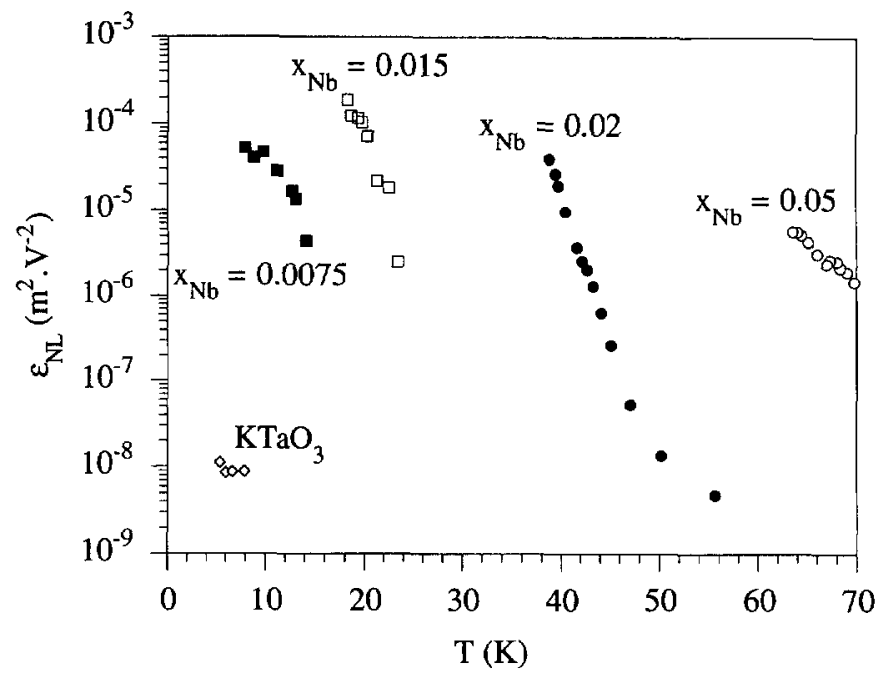

Fig. 3. - First-order non-linear susceptibility versus temperature for the five $\mathrm{KTaO}_{3}: \mathrm{Nb}$ samples. Note that the $\varepsilon_{N L}$ scale is logarithmic.

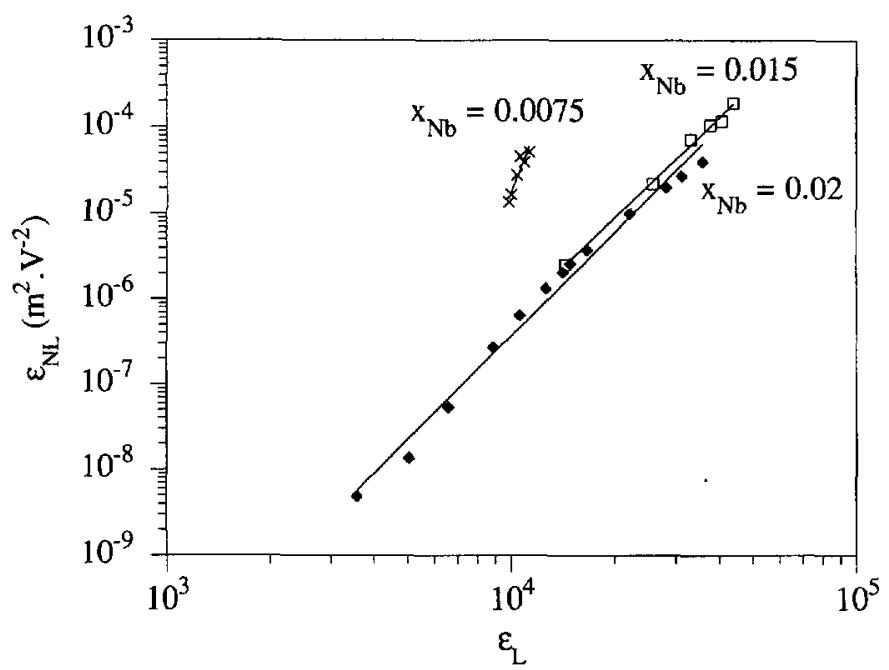

Fig. 4. - Log-log plot of the non-linear dielectric susceptibility versus the linear susceptibility for the three $\mathrm{KTaO}_{3}: \mathrm{Nb}$ samples in which $\varepsilon_{\mathrm{NL}}$ has been determined unambiguously. The solid lines are the result of the best fits to the power law $\varepsilon_{\mathrm{NL}}=K\left(\varepsilon_{\mathrm{L}}\right)^{\nu}$ (see text). The adjustable parameter $\nu\left(=\gamma_{\mathrm{NL}} / \gamma_{\mathrm{L}}\right)$ is respectively equal to $10.2\left(x_{\mathrm{Nb}}=0.0075\right), 3.82\left(x_{\mathrm{Nb}}=0.015\right)$ and $4.05\left(x_{\mathrm{Nb}}=0.02\right)$.

to the one predicted by the Landau theory $(\nu=4.05)$ suggesting that a long-range order takes place at the transition for such a $\mathrm{Nb}$ content.

In the same way, the other samples have been investigated. The main features are exhibited in Figures 3 and 4. As earlier explained, the data connected to $x_{\mathrm{Nb}}=0.05$ are only indicative but they still show a slight temperature dependent non-linearity. In fact, Figure 3 demonstrates 
that the non-linearity is extremum for the intermediate range $\left(0.0075 \leq x_{\mathrm{Nb}} \leq 0.02\right)$. For such $\mathrm{Nb}$ concentrations, $\varepsilon_{\mathrm{NL}}$ displays a behavior qualitatively very close to what has been already described for $x_{\mathrm{Nb}}=0.02$. Especially $\varepsilon_{\mathrm{NL}}$ increases strongly in the vicinity of $T_{\max }$ where values as high as $10^{-4} \mathrm{~m}^{2} \mathrm{~V}^{-2}$ are reached instead of $10^{-8} \mathrm{~m}^{2} \mathrm{~V}^{-2}$ in nominally pure $\mathrm{KTaO}_{3}$ and $10^{-6} \mathrm{~m}^{2} \mathrm{~V}^{-2}$ in $\mathrm{KTa}_{0.95} \mathrm{Nb}_{0.05} \mathrm{O}_{3}$. This can be taken as a test of disorder as will be detailed below. The fitted ratio of the non-linear and linear critical exponents yields $\nu=3.82$ for $x_{\mathrm{Nb}}=0.015$ and $\nu=10.2$ for $x_{\mathrm{Nb}}=0.0075$ (Fig. 4). For this latter sample, the poor accuracy of the $\nu$ value is to be noted due to a fit over less than one decade. Meanwhile the non-linear susceptibility diverges more strongly than it does in ferroelectrics $(\nu \gg 4)$ : this behavior seems to differ from the other tested samples suggesting a possible not purely ferroelectric phase at $T<T_{\max }$ for such a low $\mathrm{Nb}$ content.

To summarize our experimental findings in $\mathrm{KTaO}_{3}: \mathrm{Nb}$, the dielectric susceptibility $\varepsilon$ can be described analytically by an expansion of even powers of the dc field. The first nonlinear term increases on decreasing temperature, whereas extreme values are reached for $0.075 \leq x_{\mathrm{Nb}} \leq 0.02$. In these samples the $\varepsilon_{\mathrm{NL}}$ divergence is related to criticality at a temperature depending on $x$. Moreover the $\nu$ coefficient suggests that a ferroelectric phase transition occurs for $x_{\mathrm{Nb}}=0.015$ and 0.02 , i.e. at high enough concentrations to allow a long-range dipolar correlation.

\section{Discussion}

Since we have shown that equation (1) provides an accurate description of the $\mathrm{KTa}_{1-x} \mathrm{Nb}_{x} \mathrm{O}_{3}$ response, we now consider the microscopic nature of the $\varepsilon_{\mathrm{NL}}(T)$ divergence.

Same experiments have already been performed in systems displaying an analogous behavior: $\mathrm{K}_{1-x} \mathrm{Na}_{x} \mathrm{TaO}_{3}[17]$ and $\mathrm{Sr}_{1-x} \mathrm{Ca}_{x} \mathrm{TiO}_{3}$ [18]. The aim of these measurements was to study the competition between order and disorder, i.e. to get information about the question: at low impurity concentrations, do these systems freeze in a dipolar glass state or do they undergo a ferroelectric phase transition? Indeed the addition of $\mathrm{Na}^{+}$in the incipient ferroelectric $\mathrm{KTaO}_{3}$ will induce macroscopic polar distortions if $x_{\mathrm{Na}} \geq 0.12$ [21]. On the other hand, the same effect is observed by substituting $\mathrm{Ca}^{2+}$ ions in the $\mathrm{Sr}^{2+}$ site of the quantum paraelectric system $\mathrm{SrTiO}_{3}$ provided $x_{\mathrm{Ca}} \geq 0.0018$ [22]. The minimal impurity content, $x^{*}$, required for inducing a low temperature polar phase differs from one system to the others $\left(x_{\mathrm{Ca}}^{*}=0.0018\right.$ in $\mathrm{SrTiO}_{3}$, $x_{\mathrm{Nb}}^{*}=0.0075$ and $x_{\mathrm{Na}}^{*}=0.12$ in $\mathrm{KTaO}_{3}$ ). Meanwhile all of them display qualitatively the same behavior for low doping rates. Particularly in the intermediate range $\left(0.0018 \leq x_{\mathrm{Ca}} \leq 0.02\right.$, $0.0075 \leq x_{\mathrm{Nb}} \leq 0.02$ and $0.12 \leq x_{\mathrm{Na}} \leq 0.20$ ) the transition temperature $T$ is known to vary with impurity content following

$$
T \propto\left(x-x^{*}\right)^{1 / 2}
$$

instead of the linear increase observed in usual ferroelectrics.

In these systems, the dopant ions are generally thought to occupy off-center positions. Their random distribution in a highly polarizable host lattice induces electric dipoles. While the temperature is lowered, the correlation length between ionic displacements increases due to the softening of the ferroelectric phonon mode. As a consequence polar microregions start to grow around random point defects in the cubic lattice. Each off-center dopant atom is thus characterized by an effective dipole moment related to the polarization of a cloud containing the nearest neighbour unit cells. The spatial extension of theses clusters is strongly temperature dependent. This is reflected by the increase of $\varepsilon_{\mathrm{L}}(T)$ whereas $\varepsilon_{\mathrm{NL}}$, which is sensitive to the correlation between dipoles, is expected to diverge. Figure 5 displays its temperature dependence in the $\mathrm{SrTiO}_{3}: \mathrm{Ca}$ and $\mathrm{KTaO}_{3}: \mathrm{Na}$ systems from the data reported in $[18,17]$ respectively. 


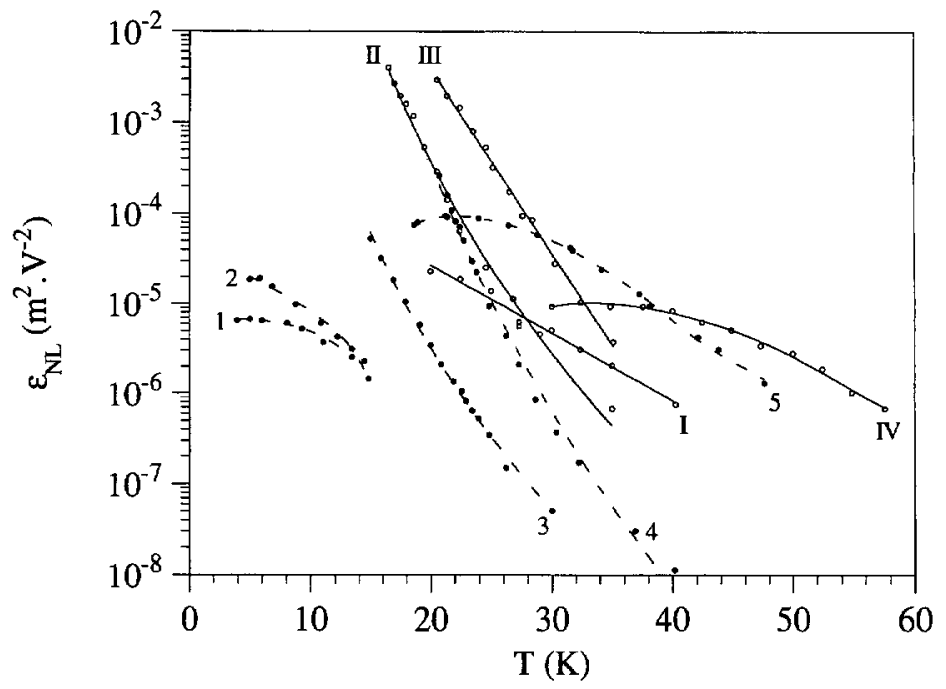

Fig. 5. - The same plot as in Figure $3\left(\varepsilon_{\mathrm{NL}}\right.$ vs. T) but for two different systems $\mathrm{K}_{1-x} \mathrm{Na}_{x} \mathrm{TiO}_{3}$ and $\mathrm{Sr}_{1-x} \mathrm{Ca}_{x} \mathrm{TiO}_{3}$, previously studied respectively in references $[17,18]$. Closed circles: $\mathrm{K}_{1-x} \mathrm{Na}_{x} \mathrm{TiO}_{3}$ with five $\mathrm{Na}$ contents (curve 1: $x_{\mathrm{Na}}=0.08,2: x_{\mathrm{Na}}=0.12,3: x_{\mathrm{Na}}=0.16,4: x_{\mathrm{Na}}=0.20$ and 5: $x_{\mathrm{Na}}=0.24$ ). Open circles: $\mathrm{Sr}_{1-x} \mathrm{Ca}_{x} \mathrm{TiO}_{3}$ (I: pure $\mathrm{SrTiO}_{3}, \mathrm{II}: x_{\mathrm{Ca}}=0.003$, III: $x_{\mathrm{Ca}}=0.004$ and IV: $x_{\mathrm{Ca}}=0.058$ ). The full and dashed lines are only guides for the eyes.

As earlier noted in $\mathrm{KTaO}_{3}: \mathrm{Nb}$ (Fig. 3), we can see that the non-linear susceptibility divergence is extreme in the intermediate range. In $\mathrm{Sr}_{1-x} \mathrm{Ca}_{x} \mathrm{TiO}_{3}$, it is three orders of magnitude stronger for $x_{\mathrm{Ca}}=0.003$ and 0.004 than in nominally pure $\mathrm{SrTiO}_{3}$ and $x_{\mathrm{Ca}}=0.058$ doped sample. These features are quite comparable with those found in the $\mathrm{Na}$ doped potassium tantalate crystals. The divergence is more clearly observed for $x_{\mathrm{Na}}=0.16$ and 0.20 than in the other samples with smaller or greater amounts of sodium. However, we point out that the cluster "seed" is very different from one system to another. In $\mathrm{KTaO}_{3}: \mathrm{Nb}$, the $\mathrm{Nb}^{5+}$ impurity ions sit at the center of the cubic oxygen octahedron while, in $\mathrm{KTaO}_{3}: \mathrm{Na}$, the $\mathrm{Na}^{+}$impurity sits outside this octahedron. Moreover in $\mathrm{SrTiO}_{3}: \mathrm{Ca}$, the $\mathrm{Ca}^{2+}$ ions are replacing $\mathrm{Sr}^{2+}$ in a tetragonal phase.

Thus, all the observations, made in three different systems in which long-range order competes with continuous random freezing, have to result from the same origin: the random dipole-dipole interaction which is doping rate dependent. This explanation, based on the development of polarized clusters, accounts for the main observed features. The cluster size, which is proportional to the inverse of the soft-mode frequency, varies hardly with temperature whereas their number is directly related to the impurities content. Such a model of interacting clusters have already been used to explain the peculiar dielectric dispersion evidenced in $\mathrm{KTa}_{0.96} \mathrm{Nb}_{0.04} \mathrm{O}_{3}$ [23]. The extreme $\varepsilon_{\mathrm{NL}}$ values, linked to correlations between dipoles and reported in the intermediate range of the three systems, results from both the numbers of clusters and their effective dipole moment, $p$.

At low doping rates, the polarized microregions can be extended to long-range due to the large distance between the two first impurity neighbours, leading to an increase of $p$. For $x<x^{*}$, i.e. when no polar phase occurs at low temperatures due to the zero-point fluctuations, the dipoles are so far from each others that they do not interact. When the impurity concentration is raised, this average distance decreases down to a threshold from which 


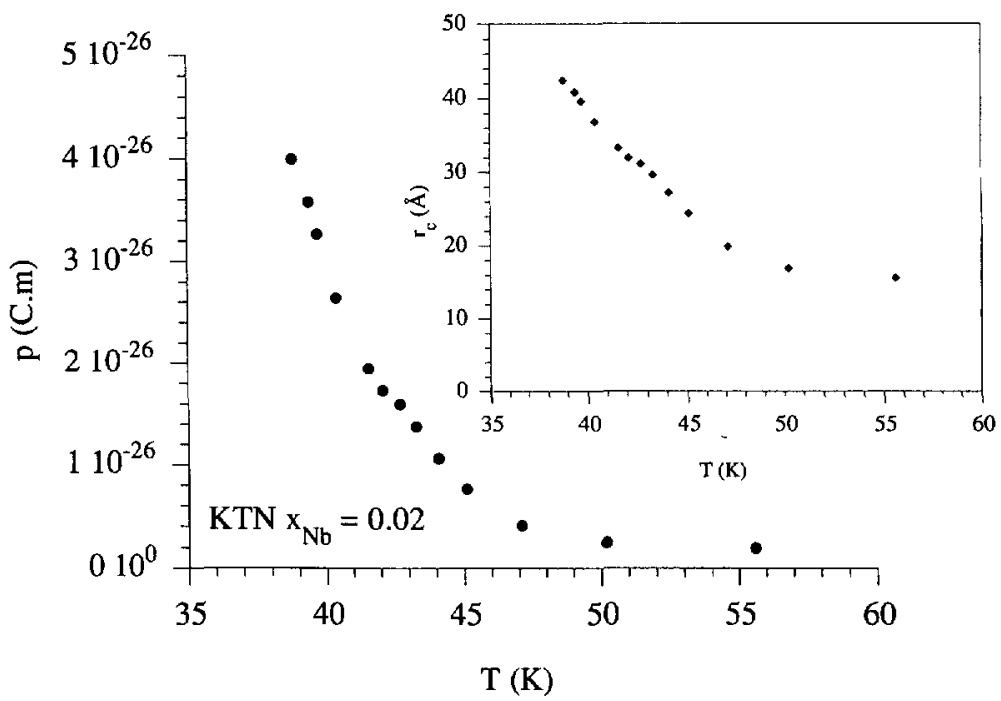

Fig. 6. - Dipolar moment as a function of temperature and calculated in a $\mathrm{KTa}_{1-x} \mathrm{NbO}_{3}\left(x_{\mathrm{Nb}}=0.02\right)$ crystal from the Langevin model. Insert: correlation radius of the distorted polarized clusters estimated from the $p$ values. In both cases, the $p$ and $r_{c}$ values at low temperatures are only indicative.

percolation takes place. For greater concentrations, the cluster number is so high that their size has to decrease and, consequently, $p$ cannot reach its maximum value. The relevant function is thus $p(x, T)$ which is however unknown. From the technique described in this paper, one can find an extremum of this function which defines the cluster percolation threshold. Away from this extremum, one can use independent clusters models, the simplest of which is the Langevin function. Within this approximation the dielectric susceptibility reads:

$$
\varepsilon(E)=\frac{N p^{2}}{3 \varepsilon_{0} k T}\left(1-\frac{p^{2}}{5 k^{2} T^{2}} E^{2}+\cdot\right)=\varepsilon_{\mathrm{L}}-\varepsilon_{\mathrm{NL}} E^{2}+\cdot
$$

From our experiments (Fig. 4), we can calculate the $\varepsilon_{\mathrm{NL}} / \varepsilon_{\mathrm{L}}$ ratio which leads to the estimated dipolar moment as a function of temperature (Fig. 6 for $x_{\mathrm{Nb}}=0.02$ ). At the highest temperature $(56 \mathrm{~K})$, we find $p=2.1 \times 10^{-27} \mathrm{C} \mathrm{m}$. The off-centered displacement of the $\mathrm{Nb}^{5+}$ and the $\mathrm{Ta}^{5+}$ in their immediate vicinity has been estimated to about $\delta \approx 0.1 \AA$ in the polarized cloud [24]. This allows to determine the size of the cluster surrounding each niobium: $r_{\mathrm{c}} \approx 15 \AA$ at $56 \mathrm{~K}$, in agreement with NMR results performed on KTN samples [9]. It is also interesting to note that radius of the same range (10-30 $\AA$ [25]) were obtained in pure [26-28] and $\mathrm{Li}$ doped [29-31] $\mathrm{KTaO}_{3}$ samples by different models [30,31] or experimental techniques (Hyper-Rayleigh [26], Raman [28], Second Harmonic [29] . ).

The simple Langevin model is valid only if the dipoles are distant from one another, i.e. provided that the electrostatic interactions between them are negligible. The $r_{\mathrm{c}}$ values found at low temperatures $(T<45 \mathrm{~K})$ are thus only indicative (insert Fig. 6). As the transition is approached, the dipoles start to influence each other and more sophisticated models have to be applied to KTN [32]. We however stress that such models neglect the chemical clustering which may result from a non-random distribution of the $\mathrm{Nb}$ ions in the $\mathrm{KTaO}_{3}$ host lattice.

To summarize, the properties observed in the intermediate range of $\mathrm{KTaO}_{3}: \mathrm{Nb}, \mathrm{KTaO}_{3}: \mathrm{Na}$ and $\mathrm{SrTiO}_{3}: \mathrm{Ca}$ can be explained by the simultaneous optimization of both the number of 
clusters and their individual size. The correlation effects between clusters, which are reflected by the $\varepsilon_{\mathrm{NL}}$ evolution, are then maximum: at these concentrations, the system is in the same time sufficiently dilute so that the cluster development is not inhibited and sufficiently doped to allow dipolar interactions. In the more niobium rich samples, these interactions may lead to the appearence of a true ferroelectric phase with long-range order due to the reorientation of the defect-centered clusters. This simple explanation, based on the temperature dependent cluster size, permits us to understand the non-linear susceptibility behavior observed in those systems without needing a glassy state to occur.

\section{Conclusion}

In the $\mathrm{Nb}$ doped $\mathrm{KTaO}_{3}$, away from the domain state, we have shown that the dielectric susceptibility is an analytical function of field. The deduced non-linear term diverges at low temperature and reaches extreme values in the intermediate range $\left(0.0075<x_{\mathrm{Nb}}<0.02\right)$. This behavior results from the polarized clusters correlations which are maximum in the vicinity of the percolation threshold. These results do not imply a dipole glass formation since the critical exponent $\nu$ is found very close to what is expected by the Landau theory for true ferroelectric phase transition.

\section{References}

[1] Shirane G., Danner H., Pavlovic A. and Pepinsky R., Phys. Rev. 93 (1954) 672.

[2] Rytz D., Thesis École Polytechnique Fédérale de Lausanne (1983).

[3] Lines M.E. and Glass A.M., Principles and Applications of Ferroelectrics and Related Materials (Oxford, Clarendon, 1977).

[4] Samara G.A., Phys. Rev. Lett. 53 (1984) 298.

[5] Kleemann W., Schäfer F.J. and Rytz D., Phys. Rev. Lett. 54 (1985) 2038.

[6] Lyons K.B., Fleury P.A. and Rytz D., Phys. Rev. Lett. 57 (1986) 2207.

[7] Chou H., Shapiro S.M., Lyons K.B., Kjems J. and Rytz D., Phys. Rev. B 41 (1990) 7231.

[8] Gehring P.M., Chou H., Shapiro S.M., Hriljac J.A., Chen D.H., Toulouse J., Rytz D. and Boatner L.A., Phys. Rev. B 46 (1992) 5116.

[9] van der Klink J.J., Rod S. and Châtelain A., Phys. Rev. B 33 (1986) 2084.

[10] Toulouse J., Di Antonio P., Vugmeister B.E., Wang X.M. and Knauss L.A., Phys. Rev. Lett. 68 (1992) 232.

[11] Bouziane E., Fontana M.D. and Kugel G.E., Ferroelectrics 125 (1992) 331.

[12] Vugmeister B.E. and Glinchuk M.D., Rev. Mod. Phys. 62 (1990) 993.

[13] Klössner A., Leitao U.A., Kleemann W. and Rytz D., Ferroelectrics 157 (1994) 245.

[14] Knauss L.A., Vugmeister B.E. and Toulouse J., Ferroelectrics 157 (1994) 251.

[15] Lyons K.B., Fleury P.A., Negran T.J. and Carter H.L., Phys. Rev. B 36 (1987) 2465.

[16] Maglione M., Höchli U.T. and Joffrin J., Phys. Rev. Lett. 57 (1986) 436.

[17] Maglione M., Thesis École Polytechnique Fédérale de Lausanne (1987).

[18] Maglione M., Lopes dos Santos M., Chaves M.R. and Almeida A., Phys. Stat. Sol. (b) 181 (1994) 73.

[19] Hemberger J., Lunkenheimer P., Viana R., Böhmer R. and Loidl A., Phys. Rev. B 52 (1995) 13159.

[20] Dec J., Kleemann W., Bianchi U. and Bednorz J.G., Europhys. Lett. 29 (1995) 31. 
[21] Davies T.G., Phys. Rev. 135 (1972) 2530.

[22] Bednorz J.G. and Müller K.A., Phys. Rev. Lett. 52 (1984) 2289.

[23] Fontana M.D., Maglione M. and Höchli U.T., J. Phys.: Condens. Matter 5 (1993) 1895.

[24] Rod S., Borsa F. and van der Klink J.J., Phys. Rev. B 38 (1988) 2267.

[25] Maglione M., Ferroelectrıcs 137 (1992) 113.

[26] Vogt H., Phys. Rev. B 41 (1990) 1184.

[27] Maglione M., Rod S. and Höchli U.T., Europhys. Lett. 4 (1987) 631.

[28] Uwe H., Lyons K.B., Carter H.L. and Fleury P.A., Phys. Rev. B 33 (1986) 6436.

[29] Azzini G.A., Banfi G.P., Giulotto E. and Höchli U.T., Phys. Rev. B 43 (1991) 7473.

[30] Stachiotti M.G. and Migoni R.L., J. Phys.: Condens. Matter 2 (1990) 4341.

[31] Vugmeister B.E. and Glinchuk M.D., Solid State Commun 48 (1983) 503.

[32] Vugmeister B.E. and Adikhari P., Ferroelectrics 157 (1994) 2625. 